
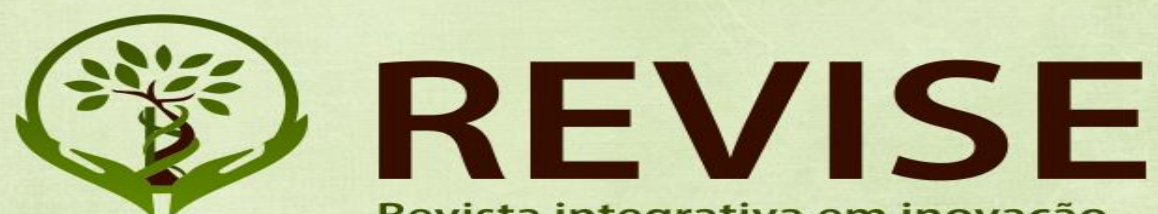

Revista integrativa em inovação tecnológica nas ciências da saúde

TETO PARA DISCUSSÃO:

\title{
INTERAÇÕES MEDICAMENTOSAS E EFEITOS ADVERSOS \\ RELACIONADOS AO USO DE FITOTERÁPICOS.
}

Renata Jesus de Souza

Eduardo Mateus dos Santos Barboza

Tiana Pereira dos Santos Cerqueira

\section{RESUMO}

UFRB

O artigo descreve a importância do cuidado no uso de medicamentos fitoterápicos e suas possíveis complicações. A metodologia de levantamento bibliográfico utilizou as bases de dados da SCIELO, MEDLINE. Através dos dados observou-se que o crescente aumento de reações adversas pelo uso de fitoterápicos é na maior parte das vezes relacionado com a falta de conhecimento da população, a automedicação utilizando esses "medicamentos" sem cautela. Diante disso torna-se evidente a necessidade da conscientização da população para o uso adequado dos fitoterápicos para que ela possa aproveitar os benefícios desses medicamentos sem por sua saúde em risco.

Palavras-Chave: Fitoterápicos. Medicamentos. Compostos químicos. Saúde.

Fitoterápicos. Revista Revise, vol 3, no Fluxo Contínuo, p.196-201. 

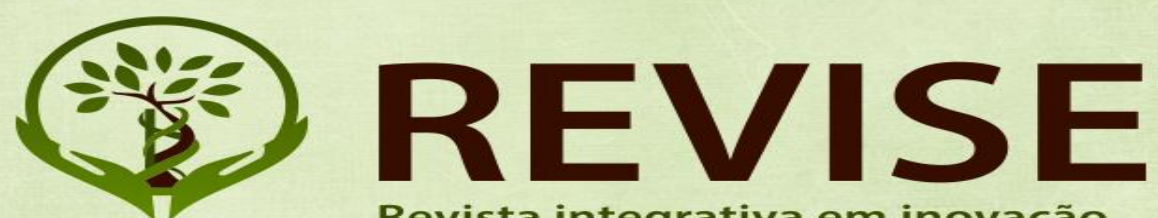

Revista integrativa em inovação tecnológica nas ciências da saúde

\title{
DRUG INTERACTIONS AND ADVERSE EFFECTS \\ RELATED TO THE USE OF PHYTOTHERAPICS.
}

\begin{abstract}
The article describes the importance of care in the use of herbal medicines and their possible complications. The bibliographic survey methodology used the databases of SCIELO, MEDLINE. Through the data it was observed that the increasing increase of adverse reactions using herbal medicines is mostly related to the lack of knowledge of the population, self-medication using these "medicines" without caution. Given this, it becomes evident the need to raise awareness of the proper use of herbal medicines so that they can enjoy the benefits of these medicines without endangering their health.
\end{abstract}

Keywords: Phytotherapics. Medicines. Chemical compounds. Cheers. 


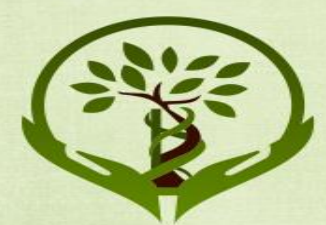

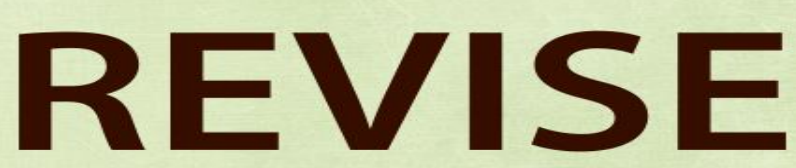

Revista integrativa em inovação tecnológica nas ciências da saúde

\section{INTRODUÇÃO}

Fitoterápicos são medicamentos que tem seus princípios ativos derivados de plantas medicinais e que tem sua eficácia e segurança comprovadas através de testes clínicos e pré clínicos fiscalizados pela ANVISA (Agência Nacional de Vigilância Sanitária). Utilizados em maior escala pela população adulta e idosa que buscam um tratamento alternativo e acreditam que os medicamentos fitoterápicos são isentos de reações adversas.

Os fitoterápicos são formados a partir de mistura de vários compostos químicos podendo então ser responsável por várias interações como sinergismos e antagonismos com outros medicamentos, muitas vezes possuindo efeitos adversos desconhecidos. $\mathrm{O}$ uso inadequado da fitoterapia pode causar toxicidade e na maioria das vezes isso ocorre quando pacientes se automedicam, mas quando se usa com cautela e orientação correta, ela pode possuir resultados terapêuticos bastante satisfatórios, contribuindo dessa forma para o bem-estar e a própria saúde do enfermo.

\section{PROBLEMA GERAL DE PESQUISA}

Descrever a importância do cuidado no uso de medicamentos fitoterápicos e suas possíveis complicações.

\section{PROBLEMA ESPECÍFICO DA PESQUISA}

Abortar o índice cada vez maior de automedicação; evidenciar as possíveis complicações do uso indiscriminado de medicamentos fitoterápicos; comparar os diferentes resultados terapêuticos entre o uso de fitoterapia em razão da terapêutica medicamentosa. 


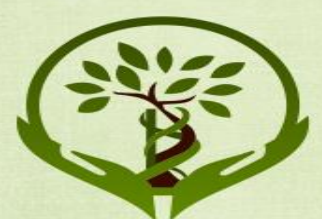

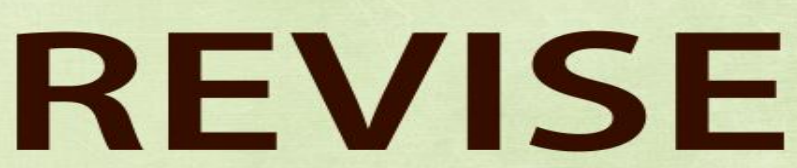

Revista integrativa em inovação tecnológica nas ciências da saúde

\section{FUNDAMENTAÇÃO TEÓRICA}

Com um número cada vez maior de estudos científicos e com os avanços na legislação brasileira e mundial, a fitoterapia se concretiza como uma opção de terapia complementar a medicina tradicional. $\mathrm{O}$ aumento do consumo de fitoterápicos pode estar relacionado ao questionamento da população sobre os perigos do uso abusivo e irracional de produtos farmacêuticos procurando substituí-los por plantas medicinais principalmente devido a comprovação da ação terapêutica e pela insatisfação da população com o sistema de saúde oficial. Algumas pessoas também necessitam do poder de controlar seu próprio corpo e recuperar sua saúde, assumindo as práticas de saúde para si ou para sua família. Os medicamentos fitoterápicos possuem um significativo destaque no mercado mundial, sendo um setor que movimenta por volta de US\$ 21,7 bilhões anualmente (CARVALHO et al,2008). O uso indiscriminado de plantas medicinais e fitoterápicos no contexto da automedicação (utilização de medicamentos sem prescrição; orientação e/o acompanhamento do médico ou dentista é alimentado pela cultura popular que facilita acesso a essas plantas e incentiva a busca do "medicamento" para grande parcela da população por um custo mais acessível (NICOLETTI et al, 2007). Os fitoterápicos são na maioria dos casos, misturas complexas de outras plantas que apresentam pouco conhecimento sobre a toxicidade e o perfil de reações adversas relacionados a qualidade do produto, adulteração, contaminação, preparação incorreta e irracional (SILVEIRA, 2007).

\section{METODOLOGIA}

Este trabalho foi elaborado a partir de um levantamento bibliográfico e revisão de literatura com busca sobre possíveis efeitos adversos e interações medicamentosas provenientes do uso da fitoterapia. Foram utilizados artigos científicos retirados das bases de dados da SCIELO, MEDLINE. 


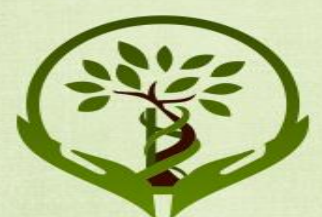

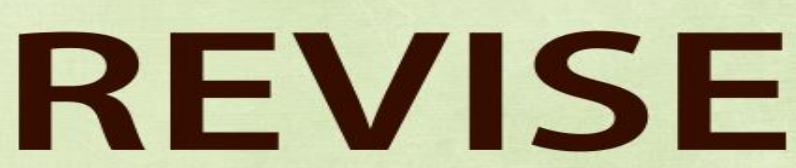

Revista integrativa em inovação tecnológica nas ciências da saúde

\section{RESULTADOS}

Através dos dados obtidos foi possível perceber que o crescente aumento de reações adversas pelo uso de fitoterápicos é na maior parte das vezes relacionado com a falta de conhecimento da população que acredita que esta é uma terapia isenta de efeitos colaterais, por se tratar na sua maior parte de composto naturais, ocasionando muitas vezes uma piora no estado de saúde do paciente, pois com essa crença, realizam a automedicação utilizando esses "medicamentos" sem cautela ou orientação.

\section{7- DISCUSSÃO}

No decorrer do presente estudo percebeu-se que a utilização dos fitoterápicos na terapia popular repercute em seu cotidiano e apesar de ser uma terapêutica "in natural" e sem dúvidas muito eficaz, este assim como qualquer outro medicamento possuem compostos químicos podendo causar vários efeitos indesejados podendo até mesmo, dependendo das suas interações, causar danos ao organismo.

A fitoterapia é um grande exemplo de como a medicina está firmemente ligada ao âmbito cultural, já que muitas vezes os fitoterápicos e seu grande "poder de cura" são indicadas por familiares a amigos ao longo das gerações, porém essa disseminação apesar de benéfica pois estimula o uso de um medicamento que traz menos risco a população pode causar sérios riscos se for utilizada de maneira inadequada como com a estimulação da automedicação.

Diante disso torna-se evidente a necessidade da conscientização da população para o uso adequado dos fitoterápicos para que ela possa aproveitar os benefícios desses medicamentos sem por sua saúde em risco. 

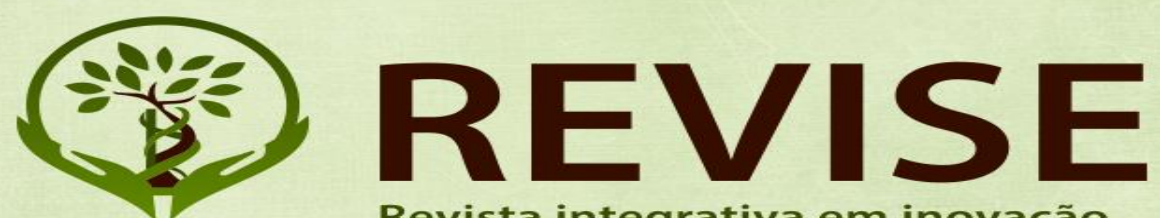

Revista integrativa em inovação tecnológica nas ciências da saúde

\section{REFERÊNCIAS}

CARVALHO, Ana CB et al. Situação do registro de medicamentos fitoterápicos no Brasil. Rev Bras Farmacogn, v. 18, n. 2, p. 314-319, 2008.

DE ANDRADE, Suzana Aparecida Lara et al. Fitoterápicos da relação nacional de medicamentos essenciais no Brasil. Revista Cubana de Plantas Medicinales, v. 22, n. $1,2017$.

ELIAS, Marcia Carlos; ALVES, Elaine. Medicina não-convencional: prevalência em pacientes oncológicos. Rev Bras Cancerol, v. 48, n. 4, p. 523-32, 2002.

NICOLETTI, Maria Aparecida et al. Principais interações no uso de medicamentos fitoterápicos. Infarma, v. 19, n. 1/2, p. 32-40, 2007.

TEIXEIRA1, João Batista Picinini; DOS SANTOS, José Vinícius; UFJF, TNC. Fitoterápicos e interações medicamentosas. Universidade Federal de Juiz de Fora, Juiz de Fora. P.1-5

TOMAZZONI, Marisa Ines; BONATO NEGRELLE, Raquel Rejane; CENTA, Maria de Lourdes. Fitoterapia popular: a busca instrumental enquanto prática terapêutica. Texto \& Contexto Enfermagem, v. 15, n. 1, 2006

Fitoterápicos. Revista Revise, vol 3, no Fluxo Contínuo, p.196-201. 